\title{
Is exercise effective treatment for osteoarthritis of the knee?
}

\author{
Robert J Petrella
}

\begin{abstract}
Objective-To review and determine the effectiveness of exercise treatment in osteoarthritis of the knee.

Methods-A computerised literature search of Medline was carried out searching between June 1966 and January 2000. Results-Twenty three randomised controlled trials were identified from the literature. Only three trials were sufficiently powered. Small to moderate beneficial effects of exercise treatment were found for pain, small beneficial effects on disability outcome measures, and moderate to great beneficial effects were observed according to patient global assessment of effect. It was not possible to obtain evidence on the content of exercise interventions, as studies were hampered by lack of attention to proper concealment, reporting of adverse effects, and long term effects of exercise treatment. The lack of standard outcomes measures is also noted. Conclusions-The available evidence indicates beneficial short term effects of exercise treatment in patients with osteoarthritis of the knee. However, the number of available studies is limited, and more research is needed to expand this recommendation. Specifically, additional trials should provide information on adherence, home based interventions, interaction with pharmacological treatments, functional outcomes measures relevant to exercise treatment in these patients, and long term effects. At present, doctors should recommend exercise to all patients with mild/moderate disease. Further study should be encouraged and exercise should be continued to be recommended as a mainstay of non-pharmacological treatment of osteoarthritis of the knee.

(Br F Sports Med 2000;34:326-331)

Keywords: osteoarthritis; knee; exercise
\end{abstract}

10 July 2000

Table 1 Medline search strategy using Medline subject headings (MeSH) and textwords (tw)

\begin{tabular}{lll}
\hline Step & Search & Results (1966-2000) \\
\hline 1 & Osteoarthritis (MeSH) and knee or arthritis (tw) & 1653 \\
2 & Exercise (MeSH) or physical training (tw) & 69 \\
3 & 1 and 2 & 23 \\
\hline
\end{tabular}

MeSH terms are assigned by Medline on the basis of subject content. Textword strategy will retrieve any article that includes the word in the title or abstract, if the abstract is included in Medline.
Persons with chronic conditions of aging such as osteoarthritis comprise a large and growing proportion of the population. ${ }^{1}$ Even though regular exercise has proven health and functional benefits, inactivity increases as patients age. Certainly, in patients with osteoarthritis, regular exercise can improve pain control, proprioception, strength, instability, and endurance, all of which improve functional independence. Until recently, however, evidence on exercise and osteoarthritis has been equivocal. ${ }^{2}$ Many retrospective studies alleged a possible negative relation between sports participation and certain occupations $\mathrm{s}^{3-5}$; however, poor study design has questioned their general applicability. This perception may have limited the use of exercise for these patients, despite published guidelines, including those of the American College of Rheumatology. ${ }^{6}$

Treatment guidelines for osteoarthritis of the knee have considered exercise as an important non-pharmacological approach. ${ }^{6}$ In addition, it directly reduces disability and corrects walking. $^{7}$

Since the publication of the above treatment

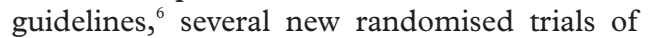
exercise treatment for osteoarthritis of the knee have been published. This paper describes the current evidence for exercise in the treatment of osteoarthritis of the knee. Efforts have been made to identify key determinants of effect including elements of the training programme, quality of the studies, and appropriateness of the outcome measures used.

\section{Methods}

WHAT MATERIALS WERE USED IN THE LITERATURE SEARCH?

A comprehensive computer assisted search of the medical, sport, and rehabilitation literature between June 1966 and January 2000 was conducted using Medline search systems. A highly sensitive search strategy of randomised controlled trials ${ }^{8}$ and systematic reviews was used. References of relevant review articles and trials were screened to identify references not contained in the main search. The search for literature was conducted using the $\mathrm{MeSH}$ headings and textwords (tw) of osteoarthritis or arthritis and knee (MeSH), exercise, or physical training (tw) (table 1). 
Table 2 Summary of selected studies on the effect of exercise on osteoarthritis of the knee

\begin{tabular}{|c|c|c|c|c|c|c|c|c|}
\hline Study & Design & Intervention group & Duration/sessions & $\begin{array}{l}\text { Duration of } \\
\text { sessions }\end{array}$ & Intensity & Pain & Disability & Walk \\
\hline 15 & RND & $\begin{array}{l}\text { 1. 15-Low Resis + education } \\
\text { 2. 5-Education }\end{array}$ & 12 weeks/3 per week & $\begin{array}{l}1 \text { hour per } \\
\text { sessions }\end{array}$ & $\begin{array}{l}3 \text { reps each } \\
\text { exercise and } \\
\text { increase to } 10 \text { at } \\
4 \text { weeks }\end{array}$ & VAS present & AIM & $\begin{array}{l}\text { Walking based on } \\
50 \% \text { performance } \\
\text { of Balke test }\end{array}$ \\
\hline 17 & RND & $\begin{array}{l}\text { 1. } 9 \text {-control sham electrical } \\
\text { stimulation } \\
\text { 2. } 9 \text {-20 min PT } 3 \text { sets of } 10 \\
\text { exercises } \\
\text { 3. } 9 \text {-education; sit to stand } \\
\text { ex, step downs }\end{array}$ & 4 weeks $/ 2$ per week & $\begin{array}{l}20 \text { min per } \\
\text { session }\end{array}$ & Not given & VAS present & $\begin{array}{l}\text { Clinical measures } \\
\text { of swelling, ROM }\end{array}$ & $\begin{array}{l}\text { Time to complete } \\
50 \mathrm{~m}\end{array}$ \\
\hline 18 & RND & $\begin{array}{l}\text { 1. } 7 \text {-Resis + diathermy } \\
\text { (hospital) } \\
\text { 2. } 7 \text {-Resis at home }\end{array}$ & 12 weeks $/ 3$ per week & Not given & Graduated Resis & No & No & $\begin{array}{l}\text { Max wt + } \\
\text { endurance }\end{array}$ \\
\hline 8 & RCT & $\begin{array}{l}\text { 1. } 144 \text {-Aerobic } \\
\text { 2. } 146 \text {-Resis (9) } \\
\text { 3. } 149 \text {-education }\end{array}$ & $\begin{array}{l}18 \text { months: } 3 \text { months } \\
\text { in-patient then } 12 \\
\text { months } \\
\text { home-based } / 3 \text { per } \\
\text { week }\end{array}$ & $\begin{array}{l}\text { 1. } 1 \text { hour } \\
\text { 2. } 1 \text { hour }\end{array}$ & $\begin{array}{l}\text { 1. } 50-70 \% \text { HRR } \\
2.1 .1 \mathrm{~kg} \text { start, } \\
\text { increase } 1-2 \text { sets } \\
\text { of } 12 \text { reps for } 3 \mathrm{~d}\end{array}$ & No & $\begin{array}{l}\text { Self-reported } \\
\text { disability score, } \\
\text { X-ray score }\end{array}$ & $\begin{array}{l}6 \text { min walk, stair } \\
\text { climbing, muscle } \\
\text { strength }\end{array}$ \\
\hline 19 & RND & $\begin{array}{l}\text { 1. Ultrasound } \\
\text { 2. Short-wave diathermy } \\
\text { 3. US + Resis } \\
\text { 4. SWD + Resis }\end{array}$ & 8 weeks $/ 3$ per week & Not given & Not given & No & No & $\begin{array}{l}\text { Functional } \\
\text { capacity; peak } \\
\text { torque }\end{array}$ \\
\hline 20 & RCT & $\begin{array}{l}\text { 1. } 47 \text {-supervised walking } \\
\text { 2. } 45 \text {-standard of care }\end{array}$ & 8 weeks/not given & Not given & Not given & Not given & AIMS & 6 min walk test \\
\hline 10 & RCT & $\begin{array}{l}\text { 1. } 40 \text {-Aerobic (walking) } \\
\text { 2. } 40 \text { - Aquatics } \\
\text { 3. } 40 \text {-ROM control ex }\end{array}$ & 12 weeks/ 3 per week & $\begin{array}{l}60 \min (30 \mathrm{~min} \\
\text { + warmup) }\end{array}$ & $\begin{array}{l}\mathrm{HR} \text { at } 60-80 \% \\
\text { max on treadmill }\end{array}$ & $\begin{array}{l}\text { AIMS, Tennessee } \\
\text { self-concept scale }\end{array}$ & Trunk flexibility & $\begin{array}{l}\text { Walking tolerance } \\
\text { on treadmill }\end{array}$ \\
\hline 12 & RCT & $\begin{array}{l}\text { 1. } 60 \text {-Home-based } 7 \\
\text { isokinetic ex exercise } \\
\text { 2. } 60 \text {-control }\end{array}$ & 8 weeks/daily & $\begin{array}{l}\text { Graduated reps } \\
\text { and sessions/day }\end{array}$ & $\begin{array}{l}\text { As per graduated } \\
\text { protocol }\end{array}$ & $\begin{array}{l}\text { WOMAC, VAS rest } \\
\text { and after ex tests }\end{array}$ & SF-36 & $\begin{array}{l}\text { Self-paced walk } \\
\text { and step test }\end{array}$ \\
\hline 21 & RND & $\begin{array}{l}\text { 1. } 7 \text {-hydrotherapy including } \\
\text { pool walking } \\
2.7 \text {-short wave diathermy }+ \\
\text { walking, cycling, step downs }\end{array}$ & 6 weeks $/ 2$ per week & $30 \mathrm{~min} / \mathrm{session}$ & Not given & VAS present & $\begin{array}{l}\text { Philadelphia } \\
\text { QOL, gait } \\
\text { analysis, ROM }\end{array}$ & Not given \\
\hline 2 & RCT & $\begin{array}{l}\text { 1. } 100 \text {-exercise tailored to } \\
\text { patient } \\
\text { 2. } 101 \text {-education }\end{array}$ & $\begin{array}{l}12 \text { weeks/ } 1-3 \text { per } \\
\text { week }\end{array}$ & $\begin{array}{l}30 \text { min per } \\
\text { session }\end{array}$ & Not given & VAS over last week & $\begin{array}{l}\text { Self-reported } \\
\text { disability, NSAID } \\
\text { use, strength }\end{array}$ & $\begin{array}{l}\text { Video of common } \\
\text { tasks, NSAID use }\end{array}$ \\
\hline
\end{tabular}

RCT, randomised controlled trial; RND, randomised not specified; No, not done; VAS, visual analogue scale; AIMS, arthritis measurement scale; WOMAC, Western Ontario McMaster pain scale; Resis, resistance exercise; Aerobic, aerobic exercise; Aquatic, water exercise; ROM, range of motion; NSAID, non-steroidal anti-inflammatory drugs; HR, heart rate; QOL, quality of life; US, ultrasound; SWD short wave diathermy.

WHAT WERE THE CRITERIA FOR STUDIES

CONSIDERED FOR INCLUSION?

Trial reports that met the following were eligible. (a) The trial concerned patients with osteoarthritis of the knee, and this was assessed using either clinical or radiological criteria (or a combination). (b) Treatment had been allocated using a randomised procedure. (c) At least one of the treatments had included exercise treatment. Exercise treatment was defined as a range of activities to improve strength, range of motion, endurance, balance, coordination, posture, motor function, or motor development. Exercise treatment can be performed actively, passively, or against resistance. ${ }^{9}$ No restrictions were made as to type of supervision or group size. Additional interventions were allowed. (d) At least one of the following measures had been included: pain, self reported disability, observed disability, patient's global assessment of effect. (e) Results had been published as a full report.

Trial reports were excluded if $(a)$ they concerned perioperative exercise treatment or (b) intervention groups received identical exercise treatment and therefore no contrast existed between intervention groups. No restrictions were made about the language of publication.

\section{Results}

WHICH STUDIES WERE SELECTED?

Sixty nine publications were initially identified (table 1). Thirty seven studies were excluded because of methodological criteria, eight stud- ies were excluded because they included review material, four were concerned with perioperative exercise treatment, and two included data reported in previous publications. Consequently, 18 publications on 17 trials (table 2) were included in this review. ${ }^{210-26}$

\section{WHAT WAS THE METHODOLOGICAL QUALITY OF} THE STUDIES?

As a consequence of the nature of exercise treatment, neither care providers nor patients can be blinded to the treatment. The most prevalent shortcomings of exercise interventions concerned co-interventions: the design of nine trials did not control for cointerventions on physical treatment strategies or medications, and in eight trials there was no report of these co-interventions. Many trials lacked sufficient information on several validity criteria: concealment of treatment allocation, level of compliance, control for cointerventions in the design, and blinding of outcome assessment.

\section{WERE THE STUDIES INFORMATIVE?}

Information on adverse effects of exercise treatment on long term (more than six months after randomisation) outcome assessment was often missing in trial reports. In three trial reports, long term follow up was mentioned but no results were presented. Other common deficiencies were in reporting on specification of eligibility criteria and description of the interventions. 
WERE THE STUDIES ADEQUATELY POWERED?

The sample size and power of the trials varied widely. Nine trials compared groups of less than 25 patients, whereas five compared more than 100 patients (median group size 39). Five studies $^{2} 10142022$ were designed with sufficient power $(>0.80)$ to detect medium sized effects. Two studies ${ }^{19} 27$ were designed with power (0.67 and 0.71 respectively) almost sufficient to detect medium sized effects.

\section{Outcomes}

IS EXERCISE TREATMENT EFFECTIVE?

Most trials identified were designed to study differences between exercise treatment and placebo treatment or no treatment. One also aimed to study differences between different exercise treatment interventions. ${ }^{17}$

WHAT ARE THE IMPORTANT OUTCOMES IN EXERCISE INTERVENTIONS?

Eight trials ${ }^{12} 17182022-25$ explicitly studied the differences between exercise interventions. Pain was assessed in all eight trials. Three outcome measures were used. In four studies, information was given on timing of pain assessment in relation to the days of exercise. In one study, ${ }^{25}$ outcome assessment preceded treatment, whereas, in another, ${ }^{26}$ pain was assessed the week after the completion of treatment. Self reported disability was assessed in five trials $^{12} 182022{ }^{24}$ and walking in four trials. ${ }^{12} 172125$

Data included assessment of aerobic walking programme, aerobic hydrotherapy, and a nonaerobic programme directed at range of motion. There was no evidence in favour of one type of exercise treatment programme over another.

PAIN

Pain was used as an outcome measure in 14 trials. In these trials, four different outcome measures were used to assess pain. No information was available on timing of the pain assessment in relation to the days of exercise. In one trial, ${ }^{17}$ data presentation was insufficient to calculate the effect size. One trial ${ }^{10}$ included two comparisons between exercise treatment interventions (aerobic exercise and resistance exercise) and a placebo treatment.

CLINICAL SETTING

In the five trials with sufficient power, ${ }^{2} 10142022$ there were differences in terms of participants and content of the intervention. Radiographic evidence indicated a mild/moderate stage of disease, and patients were recruited through doctors; community based recruitment was also used. The trial of Van Baar et al ${ }^{9}$ concerned supervised individual treatment, including strengthening exercises, range of motion exercises, and functional training over 12 weeks, while Ettinger et $a l^{10}$ used three months supervised treatment followed by a home based programme for 12 months, and Petrella and Bartha $^{14}$ and O'Reilly et $a l^{28}$ used only home based exercise. Exercises included aerobic or resistance exercises, ${ }^{210}$ while Petrella and Bartha ${ }^{14}$ used a progressive resistance programme over eight weeks. In the trials of Ettinger et $a l^{10}$ and Van Baar et $a l^{2}$ the supervised part of the intervention took 12 weeks to complete. This would seem to impose a greater burden on the care provider to deliver the programme than in the trials of Petrella and Bartha $^{14}$ and O'Reilly et al. ${ }^{28}$

Kovar et $a l^{27}$ studied two four week exercise programmes: individual weight bearing exercises and supervised group treatment consisting of non-weight bearing exercises. This study concerned patients with knee osteoarthritis for a mean duration of $>10$ years, and participants were recruited from the community and the clinic. The intervention concerned an eight week supervised group treatment that mainly consisted of "fitness walking". Other studies examined patients with knee osteoarthritis according to the criteria of the American College of Rheumatology, who were recruited from both the community and the clinic, ${ }^{15}$ and patients with knee osteoarthritis (not specified), who were recruited in the clinic, ${ }^{13}$ and included exercise interventions consisting of a 12 week walking programme ${ }^{15}$ or an eight week strength training programme monitored on a dynamometer. ${ }^{13}$

Thus the evidence indicates a small to moderate beneficial effect of exercise treatment on pain in knee osteoarthritis. This effect was found in participants with minimal to moderate osteoarthritis recruited from both community and clinic who were being treated with various types of exercise treatment for at least eight weeks.

SELF REPORTED DISABILITY

Self reported disability was measured in six trials. Three different measures were included. In one trial, ${ }^{13}$ data presentation was insufficient to calculate the effect of exercise on disability. In two trials with sufficient power, ${ }^{20}$ small effects on disability were observed.

Of the three trials with low power, ${ }^{15} 1927$ a large effect was observed in two. ${ }^{19} 27$

It can be concluded that there is evidence for a small beneficial effect of exercise on self reported disability. This effect was found in participants with minimal to moderate osteoarthritis who were recruited from both the community and the clinic and were being treated with various types of exercise.

WALKING

Walking was assessed in eight trials. In these trials, five different assessments were used. In two trials, ${ }^{13}{ }^{17}$ data presentation was insufficient to calculate the effect size.

In three trials with sufficient power, ${ }^{2}{ }^{102}$ a small beneficial effect of exercise treatment on walking performance was observed. Petrella and Bartha ${ }^{14}$ observed increased walking at self pace and self paced stepping (two measures of clinical relevance) following their exercise intervention.

In conclusion, the evidence indicates a small beneficial effect of exercise treatment on walking performance, and Petrella and Bartha ${ }^{14}$ showed a significant effect on both self selected speed of walking and stepping, both clinically relevant functional outcomes as recommended by OMERACT. ${ }^{28}$ 
PATIENT GLOBAL ASSESSMENT OF EFFECT

In only two trials was global assessment of effect by the patient used as the outcome measure. ${ }^{216}$ This indicates a need for future studies to integrate beneficial effects of exercise according to patients' global assessment.

\section{Discussion}

SUMMARY

Recent guidelines have advocated inclusion of exercise in treatment of osteoarthritis of the knee. ${ }^{6}$ However, past reports of exercise as a cause of osteoarthritis of weight bearing joints $^{3-5}$ may have reduced implementation among doctors. Further, lack of standard protocols, outcomes measures, and maintenance strategies may have also contributed to poor exercise implementation.

BACKGROUND AND RATIONALE

Two recent well designed intervention studies $^{210}$ have shown that regular physical activity in patients with osteoarthritis reduced disability; however, 18 months after the study, exercise adherence had declined by half. ${ }^{11}$ Non-participation in and withdrawal from exercise programmes remains a problem among patients with multiple chronic conditions. ${ }^{12}$ Hence programmes that are specifically designed to meet the needs of subgroups may effect long term behaviour change and exercise adoption in this population. A large randomised multicentre study by Ettinger et $a l^{10}$ showed that older patients who engage in either resistance or aerobic exercise achieved better pain control and functional outcomes at 18 months than those who only attended an educational programme. However, patients continued to take various arthritis medications while in the study, and there was no attempt to control for the class of medication. This may make decisions about inclusion of exercise difficult for practitioners.

We recently reported the effect of a brief home based progressive resistance exercise programme for patients with unilateral osteoarthritis of the knee. ${ }^{14}$ This programme consisted of a series of three exercises completed over 10 minutes a day using common household items. Compliance with the programme at two months was over $96 \%$, no adverse events were reported, and pain and physical functioning measured using a self paced walking activity increased significantly from baseline.

Despite these positive findings, no doseresponse relation between aerobic or resistance exercise and osteoarthritis has been established. In addition, issues of long term adherence and efficacy for exercise in the treatment of osteoarthritis are still unresolved.

\section{KEY FINDINGS}

Seventeen randomised controlled trials of the effectiveness of exercise treatment in osteoarthritis of the knee were assessed. It can be concluded that exercise is effective in patients with osteoarthritis of the knee. Available evidence indicates beneficial effects on all studied outcome measures: pain, self reported disability, observed disability in walking, self selected walking and stepping speed, and patient global assessment of effect. Effect size values indicated small effects on both disability outcome measures, a small to moderate effect on pain, and moderate to large effect according to patient global assessment of effect. As pain and disability are the main symptoms in patients with osteoarthritis, exercise treatment seems to be indicated.

\section{Patient type}

- Mild/moderate osteoarthritis

- Contemplating physical activity (contemplative stage of readiness)

- Impaired function, pain, and stiffness but not severe

- Associated co-morbidities that would benefit from exercise-for example, mild obesity

It is notable that conclusions are based on a small number of studies. Only five randomised controlled trials had sufficient power. ${ }^{2} 10142022$ Furthermore, trials often did not include all relevant outcome measures, especially with regard to observed disability-that is, walking - and a patient's global assessment of effect. In addition, a number of different instruments have been used for the assessment of specific outcome measures. The recently published list of candidate instruments provided by Bellamy ${ }^{23}$ can be seen as a first step to the accomplishment of standardisation of assessment.

\section{Exercise type}

- Aerobic (with or without resistance) exercise

- Fit principles (frequency: three times or more a week; intensity: mild/moderate, such as walking or weight bearing resistance; training duration: at least eight weeks for results but should be encouraged as a "life time" behavioural change)

- Use standard outcomes measures

- Counsel in clinic, but use allied health staff such as physiotherapists and kinesiologists as needed

Minimal information is available on long term effects of exercise treatment on osteoarthritis of the knee. This lack of information about long term effects is a remarkable omission, as the clinical impression is that the effects disappear over time.

There is insufficient evidence to draw conclusions on the optimal content of an exercise treatment intervention. The three trials with sufficient power showed beneficial effects of different types of exercise treatment: aerobic exercise, resistance exercise, and mixtures of several types of exercise treatment. ${ }^{21014}$ Results from trials comparing effects of different exercise treatment programmes are inconclusive. ${ }^{121718}$ 
Methodological assessment showed some major threats to the validity of clinical trials of exercise treatment. Blinding of providers and patients was absent in all studies. As a consequence of the nature of exercise treatment, blinding of both providers and patients is not possible. Therefore blinding of outcome measures is vital. However, in only half of the trial reports was blinding of outcome assessment explicitly reported. Another potential source of bias is the commonly occurring absence of information on adherence to the intervention. This hampers the interpretation of a study with negative results. It remains unclear whether the exercise treatment intervention was ineffective because of the intervention itself or the participants' failure to adhere to the treatment.

\section{Key findings}

- Exercise is indicated for patients with mild/moderate osteoarthritis of the knee but limited studies are available

- Standard interventions and outcomes measures are needed

- Doctors should stress behaviour change to engage long term benefit

- Long term efficacy has not been established

- Strategies to promote exercise adoption for general health should be the goal of doctors and their patients

In conclusion, the available evidence indicates beneficial short term effects of exercise treatment in patients with osteoarthritis of the knee. Given the limited number of studies available, this conclusion applies to patients with mild to moderate osteoarthritis recruited from both outpatient settings and the community. Beneficial effects have been found for various types of exercise treatment and recommended for patients with mild to moderate osteoarthritis of the knee. Doctors should promote physical activity in their patients with osteoarthritis of the knee. Exercise can improve symptoms, potentiate concomitant medications, and improve health in general.

Further research could expand these findings. In particular, additional clinical trials are needed to study the long term effectiveness of exercise treatment. In the design and conduct of these trials, specific attention should be paid to a sufficient sample size, adherence to exercise treatment, controls for co-interventions, blinded outcome assessment, and adequate data analysis including an intention to treat analysis. The incorporation of a standard set of outcome measures ${ }^{29} 30$ in combination with the adoption of a standard for reporting of results ${ }^{31}$ will greatly enhance evidence synthesis in this area.

1 Felson DT, Naimark A, Anderson J, et al. The prevalence of knee osteoarthritis in the elderly: the Framingham Osteoarthritis Study. Arthritis Rheum 1987;30:914-98.
2 Van Baar ME, Dekker J, Oostendorp RAB, et al. The effectiveness of exercise therapy in patients with osteoarthritis of the hip or knee: a randomized clinical trial. 7 Rheumatol the hip or knee:

3 Armstrong SJ, Read RA, Ghosh P, et al. Moderate exercise exacerbates the osteoarthritic lesion produced in cartilage by menisectomy. Osteoarthritis Cartilage 1993;1:89-96.

4 Kujala UM, Kethunen J, Puananen H. Knee osteoarthritis in former runners, soccer players, weight lifters and shooters. Arthritis Rheum 1995;38:539-46.

5 Spector T, Harris PA, Hart DJ. Risk of osteoarthritis associated with long-term weight bearing sports. Arthritis Rheum 1996;39:988-95.

6 Hochberg MC, Altman RD, Brandt KD, et al. Guidelines for the medical management of osteoarthritis. Part II. Osteoarthritis of the knee. Arthritis Rheum 1995;38:15416.

7 Dekker J, Mulder PH, Bijlsma JWJ, et al. Exercise therapy in patients with rheumatoid arthritis and osteoarthritis: a review. Advances in Behaviour Research Therapy 1993;15: 211-38.

8 Greenlaugh T. Papers that summarise other papers (systematic reviews and meta-analyses). BMf 1997;315:672-5.

9 Van Baar ME, Assendelft WJ, Dekker J, et al. Effectiveness of exercise therapy in patients with oateoarthritis of the hip and knee: a systematic review of randomized clinical trials. Arthritis Rheum 1999;42:1361-9.

10 Ettinger WH, Burns R, Messier SP, et al. A randomized trial comparing aerobic exercise and resistance exercise with a health education program in older adults with knee osteoarthritis. FAMA 1997;277:25-31.

11 Messier SP, Thompson CD, Ettinger WH. Effects of long-term aerobic or weight training regimens on gait in an older, osteoarthritic population. F Appl Biomech 1997;13: 205-25.

12 Minor MA, Hewett JE, Webel RR, et al. Efficacy of physical conditioning exercise in patients with rheumatoid arthritis and osteoarthritis. Arthritis Rheum 1989;32:1396-405.

13 Schilke JM, Johnson GO, Housh TJ, et al. Effects of musclestrength training on the functional status of patients with strength training on the functional status of patients

14 Petrella RJ, Bartha C. Home-based exercise therapy for older patients with knee osteoarthritis: a randomized clinical trial. $\mathcal{F}$ Rheumatol (in press)

15 Bautch JC, Malone DG, Vailas AC. Effects of exercise on knee joints with osteoarthritis: a pilot study of biologic markers. Arthritis Care Research 1997;10:48-55.

16 Borjesson M, Roberston E, Weidenhielm L, et al. Physiotherapy in knee osteoarthrosis: effect on pain and walking. Physiotherapy Research International 1996;1:89-97.

17 Callaghan MJ, Oldham JA, Hunt J. An evaluation of exercise regimes for patients with osteoarthritis. Clinical Rehabilitation $1995 ; 9: 213-18$.

18 Chamberlain MA, Care G, Harfield B. Physiotherapy in osteoarthritis of the knees. International fournal of Rehabilitation Medicine 1982;4:101-6. Cochrane Collaboration. The Cochrane Library. Cochrane controlled trial register. Oxford: Update Software, 1997

19 Jan MH, Lai JS. The effects of physiotherapy on osteoarthritic knees of females. If Formos Med Assoc 1991;90:1008-13.

20 Sylvester KL. Pilot study: investigation of the effect of hydrotherapy in the treatment of osteoarthritic hips. Clinical Rehabilitation 1989;4:223-8.

21 DeVet HC, Koudstoal J, Kwee WS, et al. Efforts to improve interobserver agreement in histopathological grading. $\mathcal{f}$ Clin Epidemiol 1995;48:869-73.

22 Mangione KK, McCully K, Gloviak A, et al. The effects of high intensity and low intensity cycle ergometry in older high intensity and low intensity cycle ergometry in older adults with knee oste

23 Rogind H, Bibow-Nielsen B, Jensen B, et al. The effects of a physical training program on patients with osteoarthritis of the knees. Arch Phys Med Rehabil 1998;79:1421-7.

24 Sullivan T, Allegrante JP, Peterson MG, et al. One-year follow up of patients with osteoarthritis of the knee who participated in a program of supervised fitness walking and supportive patient education. Arthritis Care Research 1998; 11:228-33.

25 Deyle GD, Henderson NE, Matekel RL, et al. Effectiveness of manual physical therapy and exercise in osteoarthritis of the knee. A randomized, controlled trial. Ann Intern Med 2000;132:173-81.

26 Maurer BT, Stern AG, Kinossian B, et al. Osteoarthritis of the knee: isokinetic quadriceps exercise versus an educational

27 Kovar PA, Allegrante JP, MacKenzie R, et al. Supervised fitness walking in patients with osteoarthritis of the knee. Ann Intern Med 1992;116:529-34

28 O'Reilly SC, Muir KR, Doherty M. Effectiveness of home exercise on pain and disability from osteoarthritis of the knee: a randomized controlled trial. Ann Rheum Dis 1999; 58:15-19

29 Bellamy N, Kirwan J, Boers M, et al. Recommendations for a core set of outcome measures in future phase III clinical trials in knee, hip and hand osteoarthritis: consensus develtrials in at OMERACT III 7 Rheumatol 1997:24:799-802.

30 Bellamy N. Osteoarthritis clinical trials: candidate variabs and clinimetric properties. 7 Rheumatol 1997;24:768-78.

Begg C, Cho M, Eastwood S, et al. Improving the quality of Begg $\mathrm{C}$, Cho $\mathrm{M}$, Eastwood S, et al. Improving the quality of
reporting clinical trials: the CONSORT statement. $¥ A M A$ 1996;276:637-9. 
True or false?

1 Patients with osteoarthritis of the knee are:

(a) Likely to have a history of repeated joint trauma.

(b) Likely to be younger and athletic.

(c) Poor candidates for exercise interventions.

(d) Not compliant with exercise therapy.

2 Evidence for exercise intervention in osteoarthritis of the knee supports:

(a) Improved pain control.

(b) Improved self reported disability.

(c) No adverse effects for patients.

(d) Long term benefit.

3 Problems with exercise trials in osteoarthritis of the knee include:

(a) Blinding of patients and providers.

(b) Confounding variables.

(c) Insufficient statistical power.

(d) Lack of standard outcomes and interventions.

4 Keys to a successful exercise intervention for osteoarthritis of the knee include:

(a) A clear definition of outcome.

(b) A clear consistent intervention.

(c) Behaviour change strategy to improve compliance and maintenance of effect.

(d) Control of confounding variables.

5 An ideal candidate for exercise intervention in osteoarthritis of the knee would be:

(a) Overweight 62 year old woman with non-insulin dependent diabetes mellitus and unilateral moderate osteoarthritis.

(b) A 64 year old male welder with severe bilateral osteoarthritis, constant pain with little effect of heavy analgesic use.

(c) A 55 year old male marathon runner with mild medial compartment unilateral osteoarthritis and intermittent pain, well controlled with analgesics when required.

(d) A 34 year old nurse, mother of two young children with significant family history of knee arthroplasty at an early age in both parents and a sibling, but no knee symptoms.

\section{Essay questions}

- Describe the shortcomings of exercise as treatment for osteoarthritis of the knee.

- Design an exercise intervention for a typical patient in your practice: include elements of mode - that is, aerobic/resistance - intensity, frequency, duration and follow up. What maintenance strategy would you employ to promote success in the long term? 